\title{
Single incision approach to totally extraperitoneal inguinal hernia repair
}

\author{
Jacek T. Białecki ${ }^{1}$, Maria M. Wieloch ${ }^{2}$, Krzysztof Kołomecki $^{3}$ \\ ${ }^{1}$ Department of General Surgery, Medical Center, Poznan, Poland \\ ${ }^{2}$ Department of General and Oncological Surgery, Hospital, Kutno, Poland \\ ${ }^{3}$ Department of Endocrine and General Surgery, First Chair of Endocrinology, Lodz, Poland
}

Videosurgery Miniinv 2014; 9 (2): 201-206

DOI: $10.5114 /$ wiitm.2014.41631

\begin{abstract}
Introduction: Nowadays the vast majority of inguinal hernia repairs are laparoscopically assisted. Single incision laparoscopy aims to accelerate recovery and provide a better cosmetic outcome after the operation.

Aim: To present our own modification of the totally extraperitoneal (TEP) procedure with a single incision and without a multiport, and compare the results with those obtained in the classic three-trocar TEP method.

Material and methods: The study group comprised 61 males. The first group consisted of 35 males who underwent single incision videoscopic alloplasty TEP. The second group comprised 26 males who underwent videoscopic alloplasty using the classic approach. The study assessed the time of operation, complications and postoperative pain based on the VAPS scale.

Results: The operation time in the first group was statistically longer compared with the time in the second group. The assessment of differences in postoperative pain in both the examined groups on the first and seventh day after the operation was considered statistically insignificant. In both the studied groups there was no recurrence of hernia established on the first and seventh day after the operation.

Conclusions: The reduced number of incisions in the TEP method with a single incision approach without a multiport involves a decrease in the number of postoperative scars, and thus offers a better cosmetic outcome compared with the classic TEP procedure. The time of TEP operation performed with a single incision approach without a multiport is statistically longer than the duration of the classic laparoscopic TEP procedure.
\end{abstract}

Key words: laparoscopy, totally extraperitoneal, one-incision approach.

\section{Introduction}

Laparoscopic repair of inguinal hernia has been performed since the 1990s. It was first reported by Ger et al. [1]. In 1994 only $9.7 \%$ of all inguinal hernia repairs were performed laparoscopically compared with $40 \%$ in 2009 [2]. Nowadays a vast majority of inguinal hernia repairs are laparoscopically assisted; it has become a gold standard procedure for recurrent and bilateral hernia. The method of choice for primary inguinal hernia repair is still that of Lichtenstein [3-5]. The next step in minimally invasive laparoscopic surgery techniques was single-port laparoscopy or videoscopy. The first case of single-port laparoscopic inguinal hernia repair was reported by Filipovic-Cugara et al. in 2009 [6]. The introduction of single incision laparoscopic surgery (SILS), and thus a decrease in the number of incisions needed to introduce trocars, aimed to reduce intraoperative injury and the number of complications as well as 
improve the cosmetic outcome. Currently, more and more laparoscopic procedures are performed with a single multiport: cholecystectomy, appendectomy, splenectomy, adrenalectomy or partial resection of the colon [6-14]. Single incision laparoscopy aims to accelerate recovery and provide a better cosmetic outcome after the operation [15]. Attempts at reducing the surgical incision have led to replacement of the multiport with surgical instruments introduced directly into the operative area through a single incision or puncture.

\section{Aim}

The objective of the study was to present our own modification of the totally extraperitoneal (TEP) procedure, which consists in performing the procedure with a single incision and without a multiport, and compare the results with those obtained in the classic three-trocar TEP method.

\section{Material and methods}

The study group comprised 61 males aged 26-77 years (mean age: 60 years) who underwent inguinal hernia repair. Their surgical treatment was provided at the Department of General Surgery, Medical Centre in Poznan from September 2010 to August 2012. The 61 studied patients were divided into two groups. The first group consisted of 35 males who underwent single incision videoscopic alloplasty TEP (totally extraperitoneal single incision - TEP SI). The other group comprised 26 males who underwent

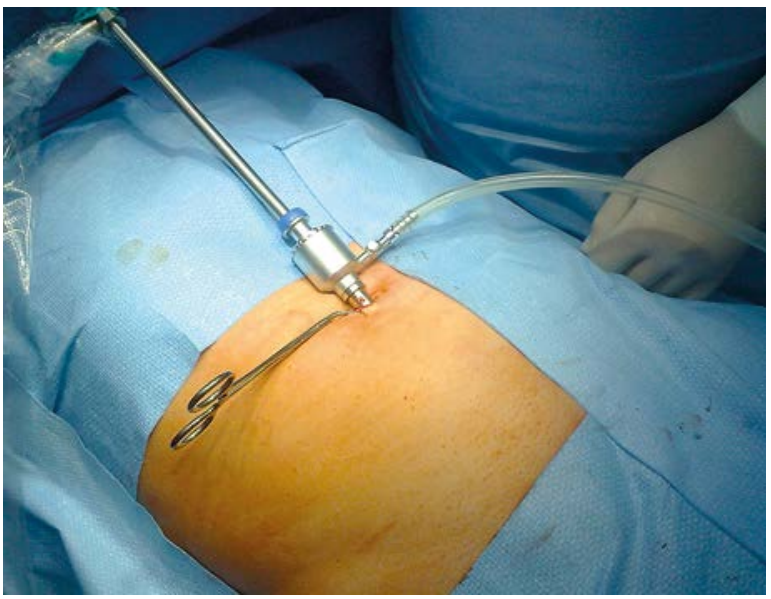

Photo 1. The transverse incision under the umbilicus with $10 \mathrm{~mm}$ trocar introduced straight into the extraperitoneal area videoscopic alloplasty using the classic approach (3 trocars). There were no significant demographic differences between the two groups. All patients were informed about the type of operation and gave their written consent to the proposed treatment. All the operations were performed under general endotracheal anaesthesia. After the standard dissection of the operative area, a transverse incision was made above the umbilicus, slightly on the side of the operated hernia $(2.7-3 \mathrm{~cm}$ in length), in both the group of patients who underwent the single incision procedure and the group of patients who had classic laparoscopy (Photo 1). The edges of the wound were retracted to the sides with Langenbeck hooks, showing the anterior surface of the rectus sheath of the rectus abdominis muscle. After the anterior rectus sheath incision was made, the medial edge of the rectus abdominis muscle was revealed, which was brushed aside, allowing the index finger to be put beneath the muscle. By moving the finger down, across the posterior surface of the rectus abdominis muscle, the preperitoneal area was dissected. Afterwards, a $10 \mathrm{~mm}$ trocar was introduced into the dissected area, $\mathrm{CO}_{2}$ was administered, and an operative camera was inserted. The preperitoneal area was dissected to the symphysis pubis toward the lateral side to the anterior superior iliac spine, by performing swinging motions with the camera. In the first group of patients, in whom the single incision procedure was performed, the next step was to insert a dissector (without trocars) through the incision below the umbilicus slightly medially to the vision trocar. In order to maintain the pneumoperitoneum in the preperitoneal area between the optic trocar and surgical tool, a packing suture was administered on the skin. The preperitoneal area dissection was continued with the help of the dissector: initially, in the midline, below the symphysis pubis, then, after finding the lower edge of the arcuate line, the area was dissected to the side. The dissection was completed after reaching the lateral wall of the iliopsoas muscle. Thereafter, by using one tool, the inguinal sac was dissected and pulled out from the inguinal canal. In the case of oblique inguinal hernia, elements of the spermatic cord were dissected free from the hernia sac; to that end, the second $5 \mathrm{~mm}$ instrument was inserted in the same way as the first one. In the case of straight inguinal hernia it was not necessary to perform parietalization because of the lack of contact between elements of the spermatic 
cord and the peritoneum. Hence, there was no need to insert the second instrument.

In the second group, after insertion of an optical instrument, the second $5 \mathrm{~mm}$ trocar was introduced in the midline, midway between the umbilicus and symphysis pubis. The dissection was continued with a dissector which was introduced through the $5 \mathrm{~mm}$ trocar. The pubic bone was exposed. Subsequently, the dissection toward the arcuate line revealed the inferior epigastric vessels and the lateral edge of the rectus abdominis muscle. An incision of the posterior rectus sheath was made, which revealed the edge of the transverse muscle. Around the anterior superior iliac spine, on the operated side, the third $5 \mathrm{~mm}$ trocar was inserted. Further dissection, consisting in the separation of spermatic cord elements from the hernia sac, was performed with the three trocars. In both the first and second group, the next step was mesh insertion. In all the 61 cases, an appropriately cut $15 \mathrm{~cm} \times 15 \mathrm{~cm}$ propylene mesh was inserted. On the inferior edge of the mesh, an arcuate incision was made which encircled iliac vessels after adapting the mesh to the preperitoneal area. The incision was also to prevent the folding of the mesh. When a required shape was achieved, the mesh was rolled in and inserted into the dissected area through the visional trocar after removing the camera. The mesh was unfolded and spread on the peritoneum in a way that prevented the mesh's edge from being located too close to the ring of the hernia. That location diminished the risk of possible recurrence of the disease. Desufflation was performed under camera supervision while observing the spreading of the peritoneum on the mesh. The mesh did not require suturing.

In both the first and second group, the same typical laparoscopic instruments were used. In the postoperative period analgesic pharmacotherapy was administered: on the day of the operation, ketoprofen $0.1 \mathrm{~g}$ twice a day; on the first day after the operation, ketoprofen $0.1 \mathrm{~g}$ once a day. All the patients were activated on the first day after the operation.

The study assessed the time of the operation, complications such as haematoma and seroma in the operated area, and recurrence of hernia during the 3-month postoperative observation period. Moreover, we assessed postoperative pain based on the visual analogue pain scale (VAPS). The VAPS is a 5-degree scale that allows for the quick and direct assessment of postoperative pain by the op- erated patient. On a 5-centimetre line, 0 indicates the absence of pain and 5 indicates the strongest pain the patient can imagine. Points between 0 and 5 , marked by the patients on the consecutive days in the postoperative period, enable easy and direct assessment of the intensity of pain and comparison of pain between examined groups. The pain was assessed based on the VAPS scale on the first and seventh day after the operation. On the first day after the operation, before discharging the patient from the Surgery Department, the postoperative wound and intensity of pain were assessed. On the seventh day after the operation, follow-up visits were scheduled during which the postoperative wound and intensity of pain were assessed based on the VAPS scale again, and sutures were removed from the wound. Subsequent follow-up visits were scheduled 3 months after the operation. During the follow-up visits, chronic pain in the operated area, incidence of complications, and recurrence of hernia were assessed. In the first group of patients, operated on using the single incision method, there was no need to convert to the classic laparoscopic TEP procedure.

\section{Results}

In the first group of patients, operated on applying the single incision method, the average operation time was $54 \mathrm{~min}$ (range: $36-82 \mathrm{~min}$ ). In the second group, operated on employing the classic TEP procedure, the average operation time was 45.27 min (range: $25-79 \mathrm{~min}$ ). The operation time in the first group was longer than the time in the second group. The difference in the operation time between the groups is statistically significant $(p=0.008)$. Figure 1 presents the average operation time in both the groups.

All the 61 studied patients were assessed for the intensity of pain based on the VAPS scale on the first and seventh day after the operation. On the first postoperative day, the average score on the VAPS scale was 1.76 points in the first group and 1.69 points in the second group, where $p=0.67$ was considered statistically significant. On the seventh day after the operation, the average score on the VAPS scale was 1.38 points in the first examined group and 1.3 points in the second group, where $p=0.56$ was considered statistically significant. The assessment of differences in postoperative pain in both the examined groups on the first and seventh day after 


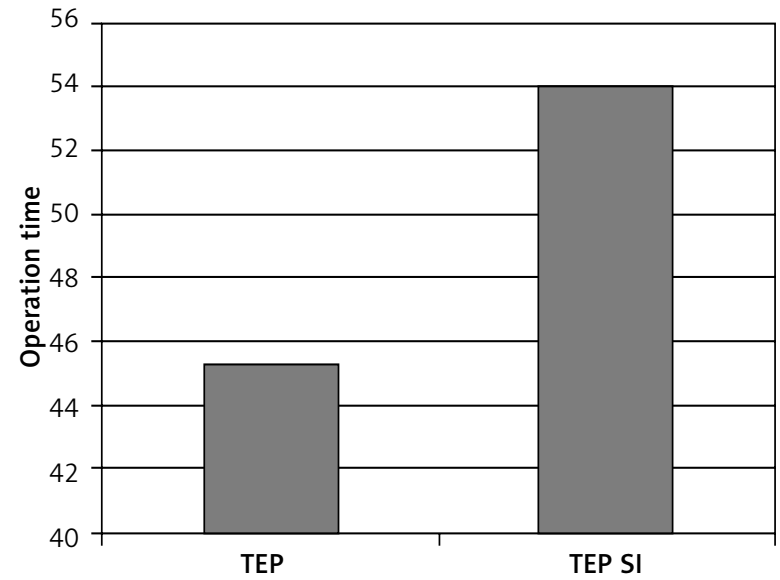

Figure 1. Comparison of the mean operation time between classic, laparoscopic TEP method and the procedure performed with a one-incision approach without using trocars

the operation was considered statistically insignificant. Figure 2 presents the assessment of postoperative pain on the first and seventh day after the operation in both the studied groups based on the average scores.

In both the studied groups of patients there was no recurrence of hernia established on the first and seventh day after the operation. The postoperative wound healing, assessed on the first and seventh day after the operation, was uncomplicated in both the first and second group. The follow-up performed three months after the operation revealed one recurrence of hernia, two haematomas, and one seroma in the area of the removed hernia sac in the first studied group. In the second group no recurrence of hernia was observed. The performed examination revealed one haematoma and two seromas in the area of the hernia sac. No case of chronic postoperative pain assessed on the VAPS scale was observed in either of the studied groups.

\section{Discussion}

The laparoscopic repair of inguinal hernia was first performed in the 1990s. Along with the development of new operative techniques, improvement in surgical instruments, and increasing surgeons' experience in laparoscopic procedures, laparoscopic inguinal hernia repair was considered a gold standard procedure in many medical centres. Due to the constant attempts to reduce intraoperative injury,

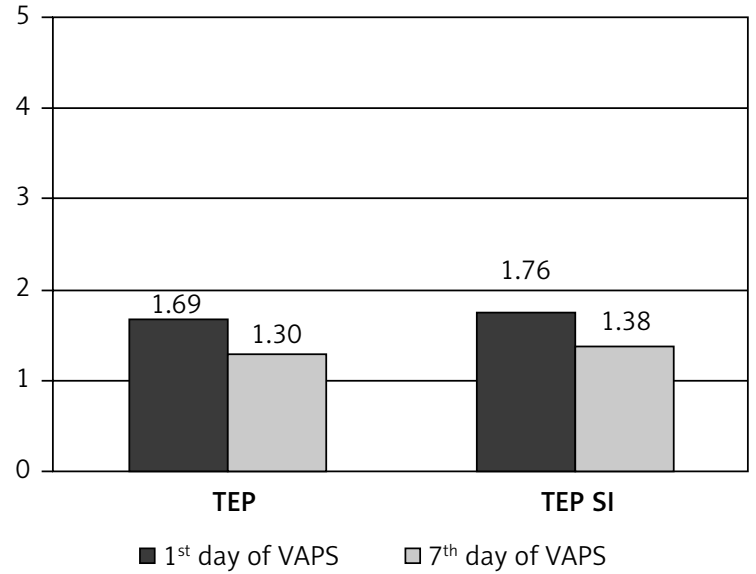

Figure 2. Assessment of the postoperative pain on the $1^{\text {st }}$ and $7^{\text {th }}$ day after the operation, in both examined groups, based on the average point values

shorten the period of convalescence, and gain the best cosmetic outcome, laparoscopic inguinal hernia repairs have also been performed using the single incision method, most often with a multiport. Totally extraperitoneal hernia repair is performed applying the classic method, using three trocars located in the abdominal cavity in a way that maintains triangulation, hence facilitating the hernia repair procedure. In many medical centres, totally extraperitoneal hernioplasty is performed with a multiport inserted through a single incision under the umbilicus. Laparoscopic procedures with a single incision approach are also performed by introducing all surgical instruments through a single incision, without a multiport. In our study, we compared operations performed employing the classic laparoscopic method with an extraperitoneal approach with operations performed applying our own modification of the TEP procedure, consisting in the introduction of surgical instruments directly into the operative area.

According to numerous literature reports, as compared with classic 3-trocar laparoscopic procedures, operations performed using single incision approach with or without a multiport may allow for diminishing the risk of complications that consist in injury to internal organs and blood vessels, and occur while instruments are being introduced into the abdominal cavity [6]. In the performed study, no complications in the form of injury to internal organs were revealed in either group of patients. Nevertheless, literature reports and long experience in laparoscopic surgery 
support the accuracy of the statement that potential complications can be reduced by decreasing the number of surgical incisions. Moreover, the reduced number of surgical incisions potentially lessens postoperative pain and diminishes the number of sites where postoperative infection may occur [6]. Replacing three incisions with one leads to a better cosmetic outcome by reducing the number of scars.

Single incision laparoscopic hernia repair makes performance of the operation more difficult as compared with the classic 3-trocar method. The loss of triangulation maintained in the classic method extends the operation time due to more technical difficulties [12]. In the study, the average operation time for operations performed employing the classic laparoscopic 3-trocar method was $45.27 \mathrm{~min}$. The average time for operations performed with a single incision was $54 \mathrm{~min}$. The difference between the first and second group is statistically significant. A lot of literature reports confirm a statistically longer operation time for procedures performed with a single incision, both with a multiport and introduction of laparoscopic instruments directly into the operated area, compared with operations performed with several incisions [12-14, 16]. Huai-Ching et al., comparing the operation time for the classic TEP method (average time: $61.8 \pm 26.0 \mathrm{~min}$ ) with the operation time for the TEP procedure performed with a single incision (average time: $61.8 \pm 26.0 \mathrm{~min}$ ), confirmed our results. As for the method using a single incision approach, apart from the statistically longer operation time, literature reports highlight a decidedly longer time required by surgeons to develop skills necessary to perform the single incision procedure correctly and safely.

The multiport use in the TEP method increases operation costs compared with procedures performed with the direct introduction of standard laparoscopic instruments into the operative area [6]. In a report comparing the classic TEP method and TEP procedure performed with a single incision approach with a multiport, $\mathrm{H}$. Tran drew attention to the difference in the operation cost, which is $\$ 150$ higher for the procedure using a multiport. Inguinal hernia repair performed applying the procedure with a single incision approach and direct introduction of standard laparoscopic instruments into the operative area eliminates additional costs of operation, simultaneously reduces operative injury, and gives a better cosmetic outcome. The introduction of lap- aroscopic instruments through a single incision directly into the operative area allows one to diminish the size of the operative incision compared with the incision necessary for multiport introduction.

The difference in pain which occurred after applying the classic laparoscopic TEP method and after the TEP procedure with a single incision approach with a multiport, assessed on the basis of the VAPS scale on the first and seventh day after the operation, did not appear to be statistically significant between the studied groups. Dąbrowiecki et al., comparing fixation of the mesh during the Lichtenstein method, did not reveal significant differences in the postoperative pain between the adhesive and penetrating method [17]. The literature reports, comparing the classic TEP method with the TEP procedure performed with a single incision approach with a multiport or with the introduction of surgical instruments directly into the operative area, indicated no differences in postoperative pain, hospitalization time, and full patient convalescence.

\section{Conclusions}

The reduced number of incisions in the TEP method with a single incision approach without a multiport involves a decrease in the number of postoperative scars, and thus offers a better cosmetic outcome compared with the classic TEP procedure. The postoperative pain, operation time, and period of full patient convalescence are comparable for the classic TEP method and TEP procedure performed with a single incision approach without a multiport. The time of TEP operation performed with a single incision approach without a multiport is statistically longer than the duration of the classic laparoscopic TEP procedure.

\section{References}

1. Ger R, Mishrick A, Hurwitz J, et al. Management of groin hernias by laparoscopy. World J Surg 1993; 17: 46-50.

2. Tran H. Safety and efficacy of single incision laparoscopic surgery for total extraperitoneal inguinal hernia repair. JSLS 2011; 15: 47-52.

3. Pielaciński K, Szczepanik A, Misiak A, et al. Randomized clinical trial comparing inguinal hernia repair with Lichtenstein technique using non-absorbable or partially absorbable mesh. Preliminary report. Videosurgery Miniinv 2011; 6: 190-206.

4. Pielaciński K, Szczepanik A, Wróblewski T. Effect of mesh type, surgeon and selected patients' characteristics on the treatment of inguinal hernia with the Lichtenstein technique. Randomized trial. Videosurgery Miniinv 2013; 8: 99-106. 
5. Bierca J, Kosim A, Kołodziejczak M, et al. Effectiveness of Lichtenstein repairs in planned treatment of giant inguinal hernia - own experience. Videosurgery Miniinv 2013; 8: 36-42.

6. Filipovic-Cugura J, Kirac I, Kulis T, et al. Single-incision laparoscopic surgery (SILS) for totally extraperitoneal (TEP) inguinal hernia repair: first case. Surg Endosc 2009; 23: 920-1.

7. Hyung Jin Kim, Jae Im Lee, Chul Lee, et al. Single-port laparoscopic appendectomy. J Korean Surg Soc 2010; 78: 338-42.

8. Tacchino R, Greco F, Matera D. Single-incision laparoscopic cholecystectomy: surgery without a visible scar. Surg Endosc 2009; 23: 896-9.

9. Bucher P, Pugin F, Morel P. Single port access laparoscopic right hemicolectomy. Int J Colorectal Dis 2008; 23: 1013-6.

10. Malladi P, Hungness E, Nagle A. Single access laparoscopic splenectomy. JSLS 2009; 13: 601-4.

11. Cywinski J, Kuzdak K, Kolomecki K. One-incision approach (SILS) for retroperitoneal videoscopicadrenalectomy. Videosurgery Miniinv 2010; 5: 70-1.

12. Tai HC, Lin CD, Chung SD, et al. A comparative study of standard versus laparoendoscopic single-site surgery (LESS) totally extraperitoneal (TEP) inguinal hernia repair. Surg Endosc 2011; 25: 2879-83.

13. Sherwinter DA. Transitioning to single-incision laparoscopic inguinal herniorrhaphy. JSLS 2010; 14: 353-7.

14. Kwon KH, Son BH, Han WK. Laparoscopic totally extraperitoneal repair without suprapubic port: comparison with conventional totally extraperitoneal repair. J Korean Surg Soc 2011; 80: 319-26.

15. Pesta W, Kurpiewski W, Łuba M, et al. Single incision laparoscopic surgery transabdominal pre-peritoneal hernia repair case report. Videosurgery Miniinv 2012; 7: 137-9.

16. Cugura JF, Kirac I, Kulis T, et al. Comparison of single incision laparoscopic totally extraperitoneal and laparoscopic totally extraperitoneal inguinal hernia repair: initial experience. J Endourol 2012; 26: 63-6.

17. Dąbrowiecki S, Pierściński S, Szczęsny W. The Glubran 2 glue for mesh fixation in Lichtenstein's hernia repair: a double-blind randomized study. Videosurgery Miniinv 2012; 7: 96-104.

Received: 4.08.2013, accepted: 14.10.2013. 